\title{
Improving Technical Communication with a Cue Awareness Intervention using Poster Presentations
}

\author{
Alcwyn Parker \\ Games Academy \\ Falmouth University \\ Cornwall, UK \\ alcywn.parker@falmouth.ac.uk
}

\author{
Gareth Lewis \\ Games Academy \\ Falmouth University \\ Cornwall, UK \\ gareth.lewis@falmouth.ac.uk
}

\author{
Michael James Scott \\ Games Academy \\ Falmouth University \\ Cornwall, UK \\ michael.scott@falmouth.ac.uk
}

\begin{abstract}
It is critical that graduates be able to articulate their designs and solutions, a capability typically assessed at interview. However, some computing graduates struggle to do so, both in writing and in face-to-face contexts. Developing competence typically requires scaffolding across the curriculum. To this end, assessed poster presentations were introduced into the undergraduate Computing curriculum at Falmouth University in 2015. Each presentation followed the conclusion of each stage's practical programming project. However, the first cohort did not perform as expected This paper describes action research conducted across the following three academic years through to 2018-19. Analyses show that although students do improve over time $(d=1.41)$, progress is slow. An intervention targeting cue awareness, use of technical notation, and parsimony had a positive impact $(d=0.73)$. However, sustaining engagement was critical to its success. This shows that instruction on cue awareness, formative feedback on notation and parsimony, as well as repetition will develop students' technical communication skills. As such, conducting poster presentations at the conclusion of software development projects is recommended but subject to the need to sustain engagement.
\end{abstract}

\section{CCS CONCEPTS}

- Software and its engineering $\rightarrow$ Programming teams; $\bullet$ Social and professional topics $\rightarrow$ Software engineering education;

\section{KEYWORDS}

Assessment, Cue Awareness, Parsimony, Technical Communication, Software Architecture, Posters, Intervention, Attainment

\section{ACM Reference Format:}

Alcwyn Parker, Gareth Lewis, and Michael James Scott. 2019. Improving Technical Communication with a Cue Awareness Intervention using Poster Presentations. In Computing Education Practice (CEP '19), January 9, 2020, Durham, United Kingdom. ACM, New York, NY, USA, 4 pages. https://doi. org/10.1145/3294016.3294019

Permission to make digital or hard copies of all or part of this work for personal or classroom use is granted without fee provided that copies are not made or distributed for profit or commercial advantage and that copies bear this notice and the full citation on the first page. Copyrights for components of this work owned by others than the author(s) must be honored. Abstracting with credit is permitted. To copy otherwise, or republish, to post on servers or to redistribute to lists, requires prior specific permission and/or a fee. Request permissions from permissions@acm.org.

CEP '20, January 9, 2020, Durham, United Kingdom

( 2019 Copyright held by the owner/author(s). Publication rights licensed to Association for Computing Machinery.

ACM ISBN 978-1-4503-6631-1/19/01 ..\$15.00

https://doi.org/10.1145/3294016.3294019

\section{INTRODUCTION}

Though there isn't a broad consensus on what constitutes 'employability' skills [27, 28], it is widely agreed that educators should nurture such skills. Communication skills regularly feature in discussions pertaining to the most important of these skills (e.g., $[5,9,16,26])$. Effective communication is critical for people working in industry as it often forms the cornerstone of a successful team who can deliver a project. Misunderstood requirements and poorly communicated architectural design choices can derail a project. It should, therefore, come as no surprise that an ability to communicate well is valued by employers [1, 7, 20,23] and is often assessed at interview [17].

To this end, ways in which students' communication skills can be developed has received considerable attention from curriculum developers [24]. This literature includes a framework proposed by Etlinger in 2006 [6] centred upon the notion that the receiver must understand a message. Success, then, is bound to three critical concepts that pertain to any communication task: purpose; strategy; and audience. Whilst a large number of educational practices build upon this and other frameworks to help students develop their communication skills, many seem to be medium or context centric. For example, getting students to deliver media presentations [12] or use guidelines to support their pair programming efforts [30]. Few seem to exercise the key elements of communication strategy needed to facilitate effective technical communication.

A method of achieving this goal would be to design communication tasks in which the purpose and audience are well-defined and constrained. Consequently, students can practice their communication strategy to improve their ability to communicate the design of highly complex computing systems to specific audiences. An opportunity to do this is the use of poster presentations at the conclusion of practical projects. Notably, of the kind widely deployed by science and engineering educators [4]. Berry's [2] survey illustrates the "added versitility" that posters provide across a wide range of educational domains and evidence to support this from as early as 1985 .

However, adapting poster presentations to computing has not been without criticism. Harichandran et al [10], for example, observe a "lax" approach to developing formal guidelines to aid students in the preparation of the design of their posters. They also note a lack of guidelines on how to adapt technical communication to maintain effectiveness. The distinct contribution of this paper, being central to the intervention presented, is the notion of cue awareness and how to train students to devise strategies that aid in communication of complex technical concepts and adapt those strategies to different audiences. 


\section{BACKGROUND}

Cue awareness is the notion that students interpret cues in briefs for set tasks to guide the way in which they approach the task. Race [21] suggests this will consequently have an impact on their ability to meet the requirements of a brief. Students that actively seek cues often outperform those students who are 'cue-blind'. To readdress the balance effort must be made to continually engage the students with the brief, signposting specific requirements and communicating potential pitfalls.

There is experimental evidence which suggests these and similar approaches can help students overcome a low level of cue awareness. An intervention based on "source representation scaffolds" successfully aided the information evaluation behaviour of undergraduate biology students [13]. Framing a poster as a source of information to be evaluated, and reversing the process, it follows that students can embed these practices into their posters in order to devise their own scaffolds to support their technical communication.

A critical challenge this poses, as highlighted by Harichandran et al [10], is the quality of the available guidance. It is often the case that there is limited constructive alignment between general advice on poster design (which may address the required content and aesthetics) and how the components of the poster can be leveraged to support students achieve in aspects that are actually being measured in a specific context (i.e., technical communication skills) (see [3] for further insight into constructive alignment). Devising guidelines to this end would help students make design decisions that better support their goal of communicating more effectively, but this is a non-trivial task.

A related challenge, is that the use of guidelines can sometimes leads to a culture of "cargo cults" [14] in which students do things because they are advised to without understanding why. This is often apparent in the design choices made by students. Posters are constructed to create meaning that will support their efforts to communicate complex ideas, however, the components students choose to include often do not serve this goals. Components might be added to posters because they give a technical appearance, despite adding very little value. For example, screenshots of arbitrary excerpt of source code or spider-like UML digrams. It is for such reasons that posters by themselves may not adequately support students' communication efforts. Bespoke guidelines that make students aware of these pitfalls would, to some extent, mitigate this.

Furthermore, the process of creating and presenting research posters is often undervalued by students [15]. This is the case even when the assignment has significant weighting. This can lead to the analysis of the software design and production of the poster being left until the last minute. Thereby, reducing the impact of efforts from faculty to encourage the 'learn by doing' approach and mitigating the benefits of experimentation, practice and iteration. In order to overcome such challenges, adding points of formative assessment can help students engage in self-regulated practice, exercising their communication skills through a process of peer assessment (see $[18,19])$.

\section{CONTEXT}

The action research discussed in this paper is situated within the BSc(Hons) Computing for Games course at Falmouth University in the UK. It is typically the case that students enter the course with academic and vocational qualifications, achieving 104-120 UCAS tariff points ${ }^{1}$. Many students report they have little to no programming experience $(\sim 43 \%)$. Only half completed a qualification in computing in the secondary education context. Median age is 19 and there is a high proportion of male students $(\sim 96 \%)$. The intake has been stable, with no statistically significant differences between 2015 and 2018 in terms of descriptives like age, gender, qualifications, and prior experience.

On the course, students are expected to collaborate in a group programming project in each of the three stages of the course. As such, it is important that these students develop their technical communication skills. This ensures that they become able to work effectively as a member of the team, and that they are able to coordinate the technical aspects of a project.

In order to help students develop these technical skills, in their first year students are provided a curriculum structured around 'agile' development practice and engage with multiple practical small group exercises intended to develop their communication skills. One such exercise, situated in their Creative Computing module, is solving media computation problems [8] through pair programming using a set of guidelines designed to improve programmer communication [30]. They learn appropriate notations, such as Unified Modelling Language (UML), to describe computing systems in their Principles of Computing module as part of a sequence of assessed worksheet tasks. They also embark upon a Development Principles module which challenges students to apply tools and techniques for management, version control systems like git, and reflect on the effectiveness of their group-work. From these modules, it was anticipated that continuing to practice communication skills and engaging in reflective practice, facilitated by an academic supervisor, would enable students to apply what they had learned in future projects and overcome the challenges that they had faced previously.

These skills were measured, and students received feedback on how to improve, at the conclusion of each group project. This was inspired, to some extent, by the model and measures proposed by Rider et al [22] (also see [25]) which are used in the medical domain. Although the measures were adapted to the computing domain and the software engineering context. This formed a uni-dimensional nine-point criterion-referenced scale measuring technical communication used across all three levels. In keeping with British academic tradition, at level six a score of seven or higher denoted high achievement and 3.5 is needed to pass.

Examining those who started in 2015, it seemed many had not improved their scores between levels, whilst others demonstrated only marginal improvement. This became worrisome, as although students successfully passed and progressed, attainment at level six was lower than anticipated $(\bar{x}=4.50, \sigma=1.29)$. In an attempt to overcome this challenge, an intervention was devised to improve the communication skills of the subsequent cohorts.

${ }^{1}$ See https://www.ucas.com for a more detailed explanation of the tariff points used in the Universities and Colleges Admission System (UCAS). 


\section{PRACTICE}

The poster presentation assignment required the student to create an $\mathrm{A} 3$ poster that supported their efforts to:

- Outline the core features of the solution;

- Illustrate their individual component in the solution;

- Provide justification for relevant design decisions.

The original intervention introduced in 2015 offered a useful medium to examine authorship, analyse and critique design decisions made by a particular student, as well as align feedback to individual strengths and weaknesses. A revised intervention was introduced in 2017 at levels five and six. This consisted of several measured steps to improve the students comprehension of and engagement with the poster assignment. The components included in this intervention are: a dedicated seminar that addresses effective communication in the context crafting demo posters, a basic template for the students to adapt, and way-points to continually engage the students with the assignment.

During the seminar students were made aware that the poster assignment carries a significant weighting and therefore, should be prioritised. Posters were championed as a vehicle for honing both oral and visual communication skills inline with industry demands. Parallels were drawn between the articulation of technical problems and their solutions in the context of programming interviews and the presentation of the demo poster. Based on suggestions from Miller [15], the $5 \mathrm{Ws} \& \mathrm{H}$ were introduced as a framework to support condensing complex problems into meaningful poster content. The students were encouraged to frame the content of the poster as a 'good story' consisting of a problem that needed to be addressed, the solution, and the impacts of the outcome. The Goldilocks Problem was summoned to illustrate the importance of providing enough content to be meaningful but not too much that it is inaccessible to the intended audience: not too big, not to small, not too complex and not too trivial.

The poster template provides a suggested structure for the demo poster and encouraged adaptation based on the specific requirements of student projects, the technical solution being address and the nature of the solution. The template has been derived from well established principles of design. The structure utilises the $80 / 20$ rule, alignment and chunking to ensure there is a clear hierarchy of information. Students are encouraged to include a variety of presentation media to facilitate a greater depth of processing for the audience. Students were provided with a list of potential pitfalls based on submissions from previous years such as: overly complex and unreadable diagrams, lack of annotation or technical explanation and charts that are not compliant with industry standards.

Finally, progress way-points across the module allowed for formative feedback and facilitated continued prompting to discourage students from leaving the assignment until the last minute. An iterative approach was championed and regular formative feedback provided.

\section{ANALYSIS}

Data were collated from 42 students who met the following inclusion criteria: (i) they provided informed consent; (ii) they submitted work for each assignment; (iii) they had attended all
Table 1: Estimated Marginal Means - Levels

\begin{tabular}{lrc}
\hline Level & Mean Score & Std Error \\
\hline 4 & 2.18 & .184 \\
5 & 3.04 & .203 \\
6 & 4.58 & .256 \\
\hline
\end{tabular}

Table 2: Estimated Marginal Means - Intervention

\begin{tabular}{lrc}
\hline Level & Mean Score & Std Error \\
\hline Intervention & 4.24 & .220 \\
No Intervention & 2.98 & .193 \\
\hline
\end{tabular}

demonstrations; and (iv) there was no missing data in their record (e.g., they were not a direct entry student). As the participants completed multiple assignments of this type over the duration of the course, there were 99 observations in the dataset from these 42 students, with graduates having at most three observations. Scores were taken directly from the marking spreadsheets and were based solely on the assessment criterion related to technical communication skill.

The data were analysed in SPSS version 20. A repeated-measures linear mixed-effects model was defined using the MIXED command. The dependent variable was [Score]. Related observations (i.e., the same student) were identifiable only by an arbitrary reference in the [Student] field. Repeated measures were conducted across [Level] of study, and the diagonal repeated covariance type was selected. The independent variables [Level], [Intervention], and their interaction [Level * Intervention] were included in the model as fixed effects. The cohort to which a student belonged, identified by [Year_of_Entry], was included in the model as a random effect.

The Type III tests of fixed effects revealed statistically significance for [Level] $(F=17.119, d f=39.131, p<.001)$ and [Intervention] $(F=8.157, d f=47.584, p=.006)$. Examination of the estimated marginal means provides some insight into the size of the effects in reference to the scale. Cohen's $d$ is calculated from these estimated marginal means using Taylor's approach [29]. Table 1 shows that students improve across levels, improving significantly between levels four and six $(d=1.41)$. Table 2 shows that the intervention had an overall positive effect on attainment $(d=0.74)$. Table 3 reveals that there were no statistically significant interaction effects $(F=0.018, d f=47.584, p=.893)$. However, given there was no intervention at level four, the lower scores at level four exaggerate the variance of the 'no intervention' group. Calculating the effect size using the standard deviations pooled by level separately is therefore sensible. This reveals the effect of the intervention is larger at level five $(d=0.58)$ than at level six $(d=0.48)$. All such pairwise comparisons in Table 3 are statistically significant at the $p<.01$ level with a Bonferroni correction.

\section{CONCLUDING REMARKS}

The use of poster presentations has been explored and developed through a process of action research. It is the nature of such research to be observational and quasi-experimental. This means that confounding factors cannot be ruled out. Nevertheless, the 
Table 3: Estimated Marginal Means - Level*Intervention

\begin{tabular}{llrc}
\hline Level & Intervention & Mean Score & Std Error \\
\hline 4 & Intervention & - & - \\
& No Intervention & 2.18 & .184 \\
5 & Intervention & 3.49 & .331 \\
& No Intervention & 2.58 & .210 \\
6 & Intervention & 4.99 & .273 \\
& No Intervention & 4.17 & .408 \\
\hline \multicolumn{2}{|l}{ Note: The intervention has not been deployed at Level 4. }
\end{tabular}

available evidence indicates that, assuming all else equal, students are successfully developing their technical communication skills using this approach. Compared to the baseline measure made at the conclusion of Level 4, the effect size across the duration of the course is very large. Likewise, the cue awareness intervention has an effect size that is greater than Hattie's "hinge point" $(d>0.4)$ [11] indicating educational relevance. Given there were no other substantial changes to the way in which poster demonstrations were taught, practiced, and assessed, and that there was no evidence to suggest that the cohorts were dissimilar, it is more likely than not that the effect is attributable to the new practice.

The main benefits of the practice are focusing students' attention on the most important elements of their design or solution to communicate, ensuring parsimony in the posters to provide maximum support without overwhelming them with extraneous information. Nurturing a culture of practice through peer review and formative feedback also seems to encourage better designs which consequently better support the communication of a solution. However, there remains scope for improvement. Even with the intervention, few students achieved high scores $(\bar{x}=4.9, \sigma=1.68)$. This seems to be, in part, because the trajectory of improvement seems to be mediated by engagement-the course team noted that increases in score in the intervention group seemed to be higher for students who had already done well at earlier levels: those presumed to be highly engaged. Those adopting the practice should therefore consider approaches to sustaining engagement.

Unfortunately, it was not possible to confirm observation about engagement without deobfuscating the data to add a measure of engagement and increasing the sample size to yield greater statistical power. However, this will be a consideration in future research on this intervention. Another limitation of this work is that it is focused on students' technical communication skills There are many other aspects of communication, notably emotional intelligence and diplomacy, which are pertinent to success at interview. Such aspects were not explored in this study. Furthermore, work showing the link between performance in poster demonstrations, performance at interview, and other relevant factors which require intervention remains ongoing.

\section{REFERENCES}

[1] Archer, W., AND DAvison, J. Graduate employability: what do employers think and want?, 2008. https://aces.shu.ac.uk/employability/resources/0802grademployability.pdf.

[2] Berry, J., AND Houston, K. Students using posters as a means of communication and assessment. Educational Studies in Mathematics 29, 1 (1995), 21-27.

[3] Biggs, J. Enhancing teaching through constructive alignment. Higher education 32, 3 (1996), 347-364.
[4] Chan, V. Teaching oral communication in undergraduate science: Are we doing enough and doing it right?. Fournal of learning design 4, 3 (2011), 71-79.

[5] Clokie, T. L., AND Fourie, E. Graduate employability and communication competence: Are undergraduates taught relevant skills? Business and Professional Communication Quarterly 79, 4 (2016), 442-463.

[6] Etlinger, H. A. A framework in which to teach (technical) communication to computer science majors. In ACM SIGCSE Bulletin (2006), vol. 38, ACM, pp. 122126

[7] Fernandez-Sanz, L. Personal skills for computing professionals. Computer 42, 10 (2009), 110-111.

[8] Guzdial, M. Exploring hypotheses about media computation. In Proceedings of the ninth annual international ACM conference on International computing education research (2013), ACM, pp. 19-26.

[9] Hanna, P., Allen, A., Kane, R., Anderson, N., McGowan, A., Collins, M., And Hutchison, M. Building professionalism and employability skills: embedding employer engagement within first-year computing modules. Computer Science Education 25, 3 (2015), 292-310.

[10] Harichandran, R. S., Nocito-Gobel, J., Brisart, E., Erdil, N. O., Collura, M. A., Daniels, S. B., Harding, W. D., and Adams, D. J. A comprehensive engineering college-wide program for developing technical communication skills in students. In 2014 IEEE Frontiers in Education Conference (FIE) Proceedings (2014), IEEE, pp. 1-8.

[11] Hattie, J. Visible learning: A synthesis of over 800 meta-analyses relating to achievement. routledge, 2008.

[12] Havill, J. T., ANd Ludwig, L. D. Technically speaking: fostering the communication skills of computer science and mathematics students. In $A C M$ SIGCSE Bulletin (2007), vol. 39, ACM, pp. 185-189.

[13] Kim, S. M., And Hannafin, M. J. The effects of source representation and goal instructions on college students' information evaluation behavior change. Computers in Human Behavior 60 (2016), 384-397.

[14] McConnell, S. Cargo cult software engineering. IEEE Software, 2 (2000), 11-13.

[15] Miller, J. E. Preparing and presenting effective research posters. Health Services Research 42,1p1 (2007), 311-328.

[16] Misra, R. K., AND Khurana, K. Employability skills among information technology professionals: A literature review. Procedia computer science 122 (2017), 63-70.

[17] Mongan, J., Kindler, N. S., And Gigut̀re, E. Programming interviews exposed: secrets to landing your next job. John Wiley \& Sons, 2012.

[18] Nicol, D. The foundation for graduate attributes: Developing self-regulation through self and peer assessment. The Quality Assurance Agency for Higher Education. Scotland (2010).

[19] Nicol, D., Thomson, A., And Breslin, C. Rethinking feedback practices in higher education: a peer review perspective. Assessment \& Evaluation in Higher Education 39, 1 (2014), 102-122.

[20] Osmani, M., Weerakkody, V., Hindi, N., ANd Eldabi, T. Graduates employability skills: A review of literature against market demand. Fournal of Education for Business (2018), 1-10.

[21] Race, P. The lecturer's toolkit, 3 ed. Routledge, 2007.

[22] Rider, E. A., Hinrichs, M. M., And Lown, B. A. A model for communication skills assessment across the undergraduate curriculum. Medical teacher 28, 5 (2006), e127-e134.

[23] Robles, M. M. Executive perceptions of the top 10 soft skills needed in today's workplace. Business Communication Quarterly 75, 4 (2012), 453-465.

[24] Ruff, S., AND CARTer, M. Communication learning outcomes from software engineering professionals: A basis for teaching communication in the engineering curriculum. In 39th IEEE Frontiers in Education Conference (2009).

[25] Schirmer, J. M., Mauksch, L., Lang, F., Marvel, M. K., Zoppi, K., Epstein, R. M., Brock, D., AND Pryzbylski, M. Assessing communication competence: a review of current tools. Fam Med 37, 3 (2005), 184-92.

[26] Succi, C., AND CANovi, M. Soft skills to enhance graduate employability: comparing students and employers' perceptions. Studies in Higher Education (2019), 1-14.

[27] Suleman, F. Employability skills of higher education graduates: Little consensus on a much-discussed subject. Procedia-Social and Behavioral Sciences 228 (2016), 169-174.

[28] Suleman, F. The employability skills of higher education graduates: insights into conceptual frameworks and methodological options. Higher Education 76, 2 (2018), 263-278.

[29] TAYlOR, A. Standardised effect size in a mixed/multilevel model. http://www.psy.mq.edu.au/psystat/documents/standardised_effect_size in_mixed_ML_models.pdf, Mar 2014.

[30] Zarb, M., Hughes, J., AND Richards, J. Industry-inspired guidelines improve students' pair programming communication. In Proceedings of the 18th ACM conference on Innovation and technology in computer science education (2013), ACM, pp. 135-140. 